\title{
Models results Comparison of different approaches to turbulence for flow past a heated flat plate
}

\author{
Zafar Malikov ${ }^{1 *}$, Dilshod Navruzov ${ }^{1}$, and Xikmatulla Djumayev ${ }^{2}$ \\ ${ }^{1}$ Institute of Mechanics and Seismic Stability of Structures, Tashkent, Uzbekistan \\ ${ }^{2}$ Tashkent State Transport University, Tashkent, Uzbekistan
}

\begin{abstract}
This paper compares the results of the well-known SpalartAllmares (SA) model and the two-fluid model for the flow around a heated flat plate. These models represent different approaches to the problem of turbulence. The SA model is a one-parameter model and a representative of the RANS models. This model is currently the most popular and is used to solve many practical problems. The advantage of this model is that its accuracy is quite good and simple for numerical implementation. Therefore, the SA model is included in almost all the codes of the software package. The two-fluid model used in this work has been developed recently [15]. In the pioneering works, it is shown that the basis for constructing this model is the possibility of representing a turbulent flow in the form of a heterogeneous mixture of two liquids. Therefore, this model is derived from the dynamics of two liquids. In these works, it is also shown that the developed two-fluid model is able to adequately describe complex anisotropic turbulences. The fundamental difference between these two models is that the SA model uses the substance transfer equation, while the two-fluid model uses the dynamics equation. To compare the two models, we compare their numerical results with the known experimental data. It is shown that the results of both models are close to each other and are in good agreement with the experimental data.
\end{abstract}

\section{Introduction}

A large number of scientific publications are devoted to the study of heat transfer in a turbulent flow past a heated flat plate. Despite its simplicity, this task makes it possible to reveal the general laws of heat transfer in a near-wall turbulent flow. The plate longitudinally streamlined by laminar and turbulent flows is one of the most widespread surface elements of modern heat exchangers. Even the initial section of the pipe can be viewed as a plate. Therefore, the knowledge of the flow patterns of heat transfer processes on the plate, from the point of view of modern practice and the theory of heat transfer, remains highly relevant. Often, when developing exact and approximate analytical methods for solving various heat transfer problems, for the purpose of their verification, they turn to

\footnotetext{
*Corresponding author: malikov@mail.ru
} 
the flow past a flat plate, which once again confirms the importance of accurate knowledge of the patterns of its heat transfer. The results of the well-known Spalart-Allmares RANS model and a new two-fluid model for flow past a heated flat plate are compared in the article. These models represent different approaches to the problem of turbulence. It is known that the SA model is a model of the Reynolds approach, and the two-fluid model is based on the dynamics of two fluids.

\section{Materials and Methods}

Reynolds approach and the Spalart-Almares (SA) turbulence model. Let us consider a flat plate heated by turbulent air flow with a zero pressure gradient. The plane stationary motion of an incompressible fluid in the absence of mass forces and with variable physical properties is expressed by the Reynolds averaged Navier-Stokes equations [1]:

$$
\left\{\begin{array}{l}
\frac{\partial u}{\partial x}+\frac{\partial \vartheta}{\partial y}=0 \\
u \frac{\partial u}{\partial x}+\vartheta \frac{\partial u}{\partial y}=\frac{\partial}{\partial y}\left[\left(v+v_{t}\right) \frac{\partial u}{\partial y}\right] \\
u \frac{\partial T}{\partial x}+v \frac{\partial T}{\partial y}=\frac{\partial}{\partial y}\left(\left(\frac{v}{\operatorname{Pr}}+\frac{v_{t}}{\operatorname{Pr}_{t}}\right) \frac{\partial T}{\partial y}\right)
\end{array}\right.
$$

where $u, \vartheta-$ are radial and axial velocities, $v, v_{t}$ are the molecular and turbulent viscosity, $\mathrm{Pr}, \mathrm{Pr}_{t}$ are molecular and turbulent Prandtl numbers [12]. This system of equations is open (incomplete) and, to close it, various RANS turbulence models were used. For this purpose, the well-known Spalart-Allmaras turbulence model was used in this study. This model belongs to the class of one-parameter turbulence models, as only one additional equation is used to find the unknown turbulent viscosity. The practice has shown that it is a lowReynolds-number model that describes the entire flow region, including the near-wall layers. It has good stability and reliability and does not require high density computational grid [2]. The SA model has the form:

$$
u \frac{\partial \widetilde{v}}{\partial x}+\vartheta \frac{\partial \widetilde{v}}{\partial y}=P_{w}-D_{w}+\frac{1}{\sigma}\left[\frac{\partial}{\partial y}\left((v+\tilde{v}) \frac{\partial \widetilde{v}}{\partial y}\right)+C_{\sigma 2}\left(\frac{\partial \tilde{v}}{\partial y}\right)^{2}\right]
$$

Here $P_{w}$ and $D_{w}$ are both the generation and dissipation terms. Unknown functions are defined as follows:

$$
P_{w}=C_{b 1} f_{r 1}\left(1-f_{i 2}\right) \tilde{S} \widetilde{v}, D_{w}=\left[C_{w 1} f_{w}-\frac{C_{b 1}}{k^{2}} f_{i 2}\right]\left(\frac{\widetilde{v}}{d}\right)^{2}, \quad v_{t}=\widetilde{v} f_{v 1}
$$

The rest of the quantities are constants of the model and are presented in detail in [2].

New two-fluid turbulence model. A mathematical model of turbulent heat transfer based on the two-fluid dynamics is presented in detail in [3], and its application to heat transfer is given in [4]. The two-fluid model in the Cartesian coordinate system has the following form: 


$$
\left\{\begin{array}{l}
\frac{\partial U}{\partial x}+\frac{\partial V}{\partial y}=0 \\
U \frac{\partial U}{\partial x}+V \frac{\partial V}{\partial y}=\frac{\partial}{\partial y}\left(v \frac{\partial U}{\partial y}-u v\right), \\
\left.U \frac{\partial u}{\partial x}+V \frac{\partial u}{\partial y}=-\left(1-C_{s}\right) \frac{\partial U}{\partial y} v+\frac{\partial}{\partial y} v_{x y} \frac{\partial u}{\partial y}-K_{f} u\right), \\
\left.U \frac{\partial v}{\partial x}+V \frac{\partial v}{\partial y}=-C_{s} \frac{\partial U}{\partial y} u+\frac{\partial}{\partial y} 2 v_{y y} \frac{\partial v}{\partial y}-K_{f} v\right), \\
U \frac{\partial T}{\partial x}+V \frac{\partial T}{\partial y}=\frac{\partial}{\partial y}\left(k \frac{\partial T}{\partial y}-t v\right), \\
U \frac{\partial t}{\partial x}+V \frac{\partial t}{\partial y}=-\frac{\partial T}{\partial y} v+\frac{\partial}{\partial y} k_{y} \frac{\partial t}{\partial y}-K_{t} t
\end{array}\right.
$$

where,

$$
v_{x y}=3 v+2\left|\frac{u v}{\frac{\partial U}{\partial y}}\right|, \quad v_{n y}=3 v+2\left|\frac{v v}{\frac{\partial U}{\partial y}}\right|
$$

Let us reduce the system of equations to a dimensionless form. To do this, we correlate all velocities to the oncoming flow velocity $U_{\infty}$ and all distances to the length $L=v R e$ / $U_{\infty}$, and temperatures to the oncoming flow temperature $T_{\infty}$.

$$
\left\{\begin{array}{l}
\frac{\partial \bar{U}}{\partial \bar{x}}+\frac{\partial \bar{V}}{\partial \bar{y}}=0, \\
\bar{U} \frac{\partial \bar{U}}{\partial \bar{x}}+\bar{V} \frac{\partial \bar{V}}{\partial \bar{y}}=\frac{\partial}{\partial \bar{y}}\left(\frac{1}{\operatorname{Re}} \frac{\partial \bar{U}}{\partial \bar{y}}-\bar{u} \bar{v}\right), \\
\bar{U} \frac{\partial \bar{u}}{\partial \bar{x}}+\bar{V} \frac{\partial \bar{u}}{\partial \bar{y}}=-\left(1-C_{s}\right) \frac{\partial \bar{U}}{\partial \bar{y}} \bar{v}+\frac{\partial}{\partial \bar{y}} \bar{v}_{x y} \frac{\partial \bar{u}}{\partial \bar{y}}-K_{f} \bar{u}, \\
\bar{U} \frac{\partial \bar{v}}{\partial \bar{x}}+\bar{V} \frac{\partial \bar{v}}{\partial \bar{y}}=-C_{s} \frac{\partial \bar{U}}{\partial \bar{y}} \bar{u}+\frac{\partial}{\partial \bar{y}} 2 \bar{v}_{y y} \frac{\partial \bar{v}}{\partial \bar{y}}-K_{f} \bar{v}, \\
\bar{U} \frac{\partial \bar{T}}{\partial \bar{x}}+\bar{V} \frac{\partial \bar{T}}{\partial \bar{y}}=\frac{\partial}{\partial \bar{y}}\left(\frac{1}{\operatorname{Pr} \operatorname{Re}} \frac{\partial \bar{T}}{\partial \bar{y}}-\bar{t} \bar{v}\right), \\
\bar{U} \frac{\partial \bar{t}}{\partial \bar{x}}+\bar{V} \frac{\partial \bar{t}}{\partial \bar{y}}=-\frac{\partial \bar{T}}{\partial \bar{y}} \bar{v}+\frac{\partial}{\partial \bar{y}} \bar{k}_{y} \frac{\partial \bar{t}}{\partial \bar{y}}-K_{t} \bar{t},
\end{array}\right.
$$

where,

$$
\bar{v}_{x y}=\frac{3}{\operatorname{Re}}+2\left|\frac{\bar{u} \bar{v}}{\frac{\partial \bar{U}}{\partial \bar{y}}}\right|, \bar{v}_{n y}=\frac{3}{\operatorname{Re}}+2\left|\frac{\overline{v v}}{\frac{\partial \bar{U}}{\partial \bar{y}}}\right|, \bar{k}_{y}=\frac{3}{\operatorname{Pr} \operatorname{Re}}+2\left|\frac{\bar{t} \bar{v}}{\frac{\partial \bar{T}}{\partial \bar{y}}}\right|
$$


where $\operatorname{Pr}=v / \kappa$ is the Prandtl molecular number. The coefficients of friction and heat transfer have the form:

$$
\begin{gathered}
K_{f}=C_{1} \sqrt{2 C_{s}\left(1-2 C_{s}\right)}\left|\frac{\partial \bar{U}}{\partial \bar{y}}\right|+C_{2} \frac{|\bar{v}|}{\bar{y}}, \\
K_{t}=C_{t 1} \sqrt{2 C_{s}\left(1-2 C_{s}\right)}\left|\frac{\partial \bar{U}}{\partial \bar{y}}\right|+C_{t 2} \frac{\bar{\tau}_{w}}{\bar{q}_{w}}\left|\frac{\partial \bar{T}}{\partial \bar{y}}\right| .
\end{gathered}
$$

In this expression $C_{t 1}=0.939, C_{t 2}=0.1, C_{s}=0.2, C_{1}=0.7825, C_{2}=0.306$ are the empirical constants, $\tau_{w}$ is the friction stress, $q_{w}$ is the heat transfer flow of the wall.

An identical algorithm was used for the numerical implementation of the models. For this, we introduce a generalized stream function $\psi$ for which the following relations are true:

$$
u=\frac{\psi \partial \psi}{\partial y}, \quad \vartheta=-\frac{\psi \partial \psi}{\partial x}
$$

Then the equation of continuity in systems (1) and (4) will be satisfied automatically. Let us write system (1) and (4) in von Mises variables $(\xi, \psi)$ [5]. The derivatives are determined by the formulas:

$$
\frac{\partial}{\partial x}=\frac{\partial}{\partial \xi}-\frac{\vartheta}{\psi} \frac{\partial}{\partial \psi}, \frac{\partial}{\partial y}=\frac{U}{\psi} \frac{\partial}{\partial \psi} .
$$

In the new variables, the system of equations (1) and the Spalart-Allmares model have the form

$$
\left\{\begin{array}{l}
\frac{\partial u}{\partial \xi}=\frac{1}{\psi} \frac{\partial}{\partial \psi}\left[\frac{u}{\psi}\left(v+v_{t}\right) \frac{\partial u}{\partial \psi}\right] \\
\frac{\partial T}{\partial \xi}=\frac{1}{\psi} \frac{\partial}{\partial \psi}\left[\frac{u}{\psi}\left(\frac{v}{\operatorname{Pr}}+\frac{v_{t}}{\operatorname{Pr}_{t}}\right) \frac{\partial T}{\partial \psi}\right], \\
\frac{\partial \widetilde{v}}{\partial \xi}=\frac{P_{w}-D_{w}}{V_{z}}+\frac{1}{\sigma}\left[\frac{1}{\psi} \frac{\partial}{\partial \psi}\left(\frac{u}{\psi}(v+\tilde{v}) \frac{\partial \tilde{v}}{\partial \psi}\right)+C_{62} u\left(\frac{1}{\psi} \frac{\partial \tilde{v}}{\partial r}\right)^{2}\right]
\end{array}\right.
$$
form:

The system of equations of turbulence of the two-fluid model in new variables has the 


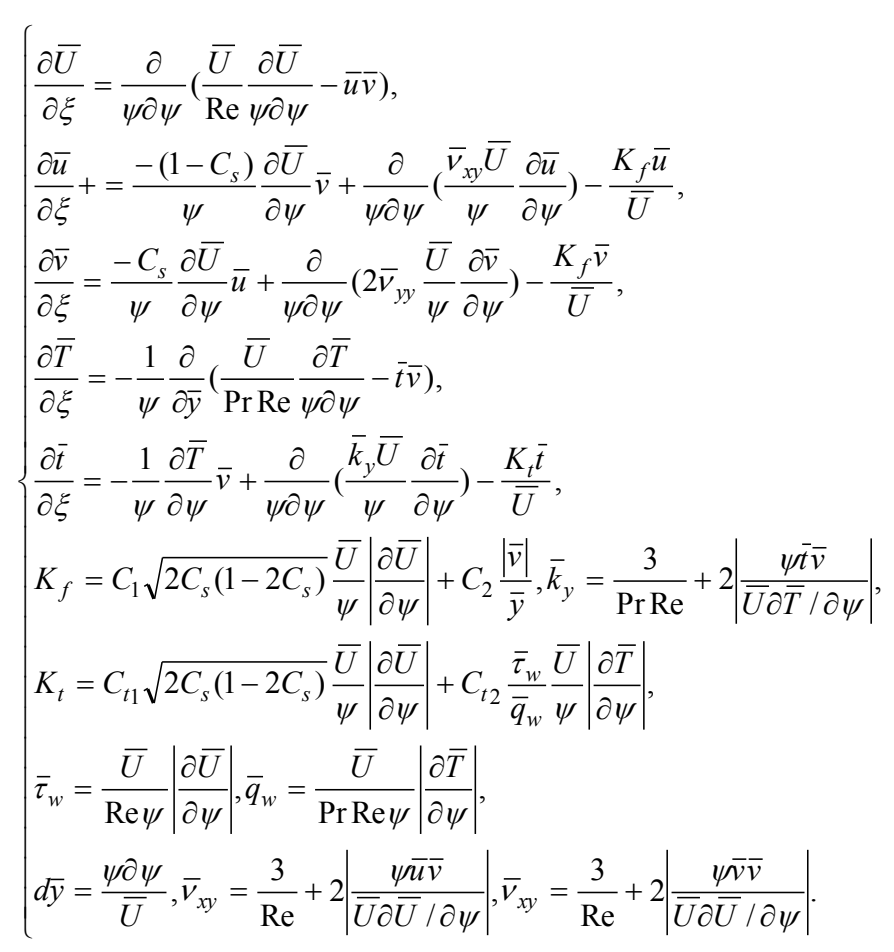

Numerical calculation of the systems of equations (6) and (7) was conducted using an implicit finite difference scheme. In the transverse direction, the center difference was used. The sweep method was used to solve the implicit scheme. The integration steps were $\Delta \xi=0.00002, \Delta \psi=0.0002$. The number of design points in the transverse direction was 500. The following boundary conditions were set for the problem: on the wall, i.e., at $\psi=0: \bar{U}=\bar{u}=\bar{v}=0$ and at $\psi \rightarrow \propto:: . \bar{U}=1, \bar{u}=\bar{v}=0$, at $\xi=0$ and $\psi=\Delta \psi$. The calculations were conducted at the Reynolds number $\mathrm{Re}=5 \cdot 10^{6}$. Thus, until the section $\xi=0$ : the flow was uniform, and then it became turbulent due to an increase in the relative velocities of the moles.

\section{Results and Discussion}

Here are some specific examples that briefly illustrate the properties of the above turbulent models. Figures $1 a, b$ and $c$ show the results of two turbulent models. They also present experimental data from [8] for the temperature profile at various Prandtl numbers. 

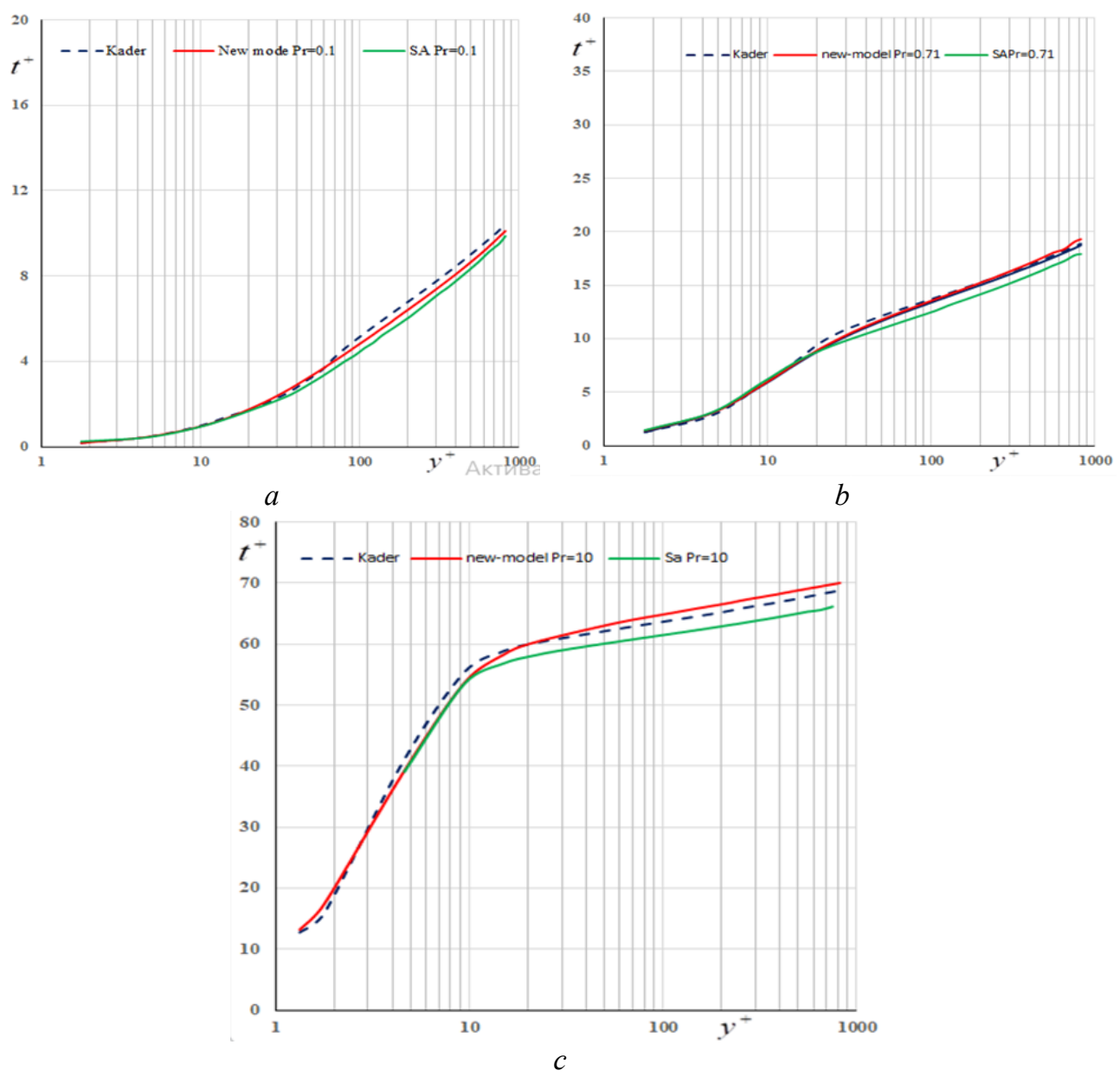

Fig. 1. $a, b$ and $c$ the results of two turbulent models

These graphs are $y^{+}=y / l_{*}, t^{+}=\frac{\left|T_{w}-T_{\infty}\right|}{t_{*}}$. As seen from these graphs, the coincidence of the numerical results with the experimental data is quite satisfactory.

Figures $2 a, b, c$ and $d$ show comparisons of the results of two turbulent models with experimental data given in [8] for the Nusselt number $N u_{x}$ at different Prandtl numbers.

It is known that the characterizing parameter of a body heat transfer is the Stanton number:

$$
S t=-\frac{1}{\Delta \bar{T} \operatorname{Pr} \operatorname{Re}}\left(\frac{\bar{U}}{\phi} \frac{\partial \bar{T}}{\partial \phi}\right)_{w}
$$

To describe the heat transfer properties of a plate in a turbulent flow, the Nusselt number $N u_{x}=\operatorname{Re}_{x} \operatorname{Pr} S t$. is a convenient parameter. 
Comparing the numerical results obtained, Figures $2 a, b, c$ and $d$ show the values of the Nusselt number from empirical formulas of Leinhard [7] and Karman [8]. The empirical expression for the Stanton number, according to Lainhard, has the form:

$$
S t=\frac{C_{f} / 2}{1+12.8\left(\operatorname{Pr}^{0.68}-1\right) \sqrt{C_{f} / 2}}
$$

and according to Karman, it is $S t=\frac{C_{f} / 2}{1+5\left(\operatorname{Pr}-1+\ln \left(1+\frac{5}{6}(\operatorname{Pr}-1)\right)\right) \sqrt{C_{f} / 2}}$.

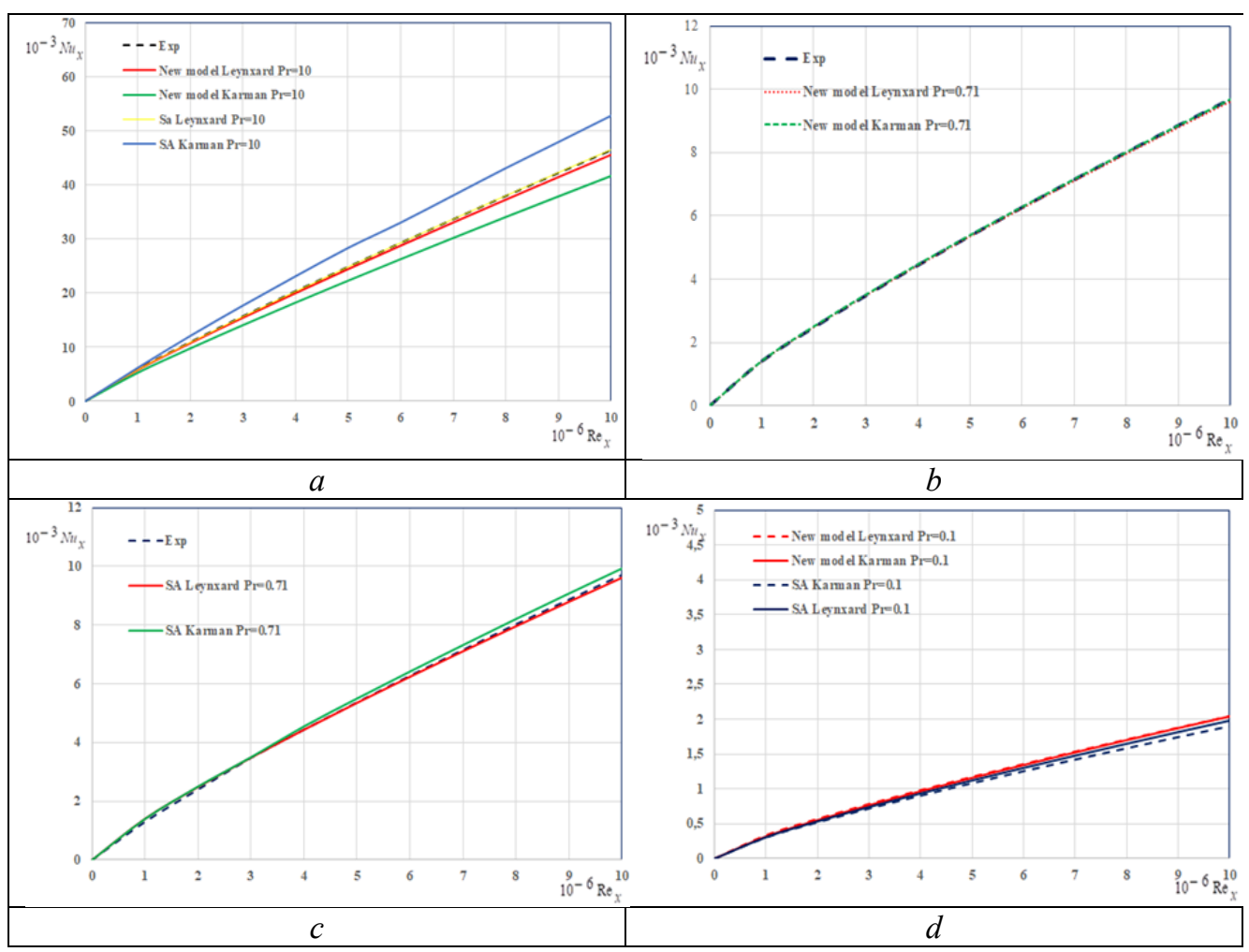

Fig. 2. $a, b, c$ and $d$ show comparisons of the results of two turbulent models with experimental data given in [8] for the Nusselt number $N u_{x}$ at different Prandtl numbers

\section{Conclusions}

The results of turbulent models of the Reynolds approach and the two-fluid approach for turbulent flow past a heated flat plate are compared in the article. The study showed that both models describe well the heat transfer process. However, the two-fluid model has the advantage of describing complex anisotropic flows for numerical implementation. Therefore, the new two-fluid model can be successfully used in more complex heat transfer problems. 


\section{References}

1. Loitsyansky L G, The mechanics of fluid and gas (M.: Science) (1987)

2. Spalart, P.R. and Allmaras S.R, A One-Equation Turbulence Model for Aerodynamics Flows, Boeing Commercial Airplane Group, Seattle, Washington. (1992)

3. Malikov Z.M, Mathematical Model of Turbulence Based on the Dynamics of Two Fluids. Applied Mathematical, (2020)

4. Malikov Z.M, Mathematical model of turbulent heat transfer based on the dynamics of two fluids. Applied Mathematical Modelling 91 pp. 186-213.(2021)

5. Mises R von Z Angew Math Mech. 7. pp 425-431 (1927)

6. Kader B.A, Temperature and concentration profiles in fully turbulent boundary layers. International Journal of Heat and Mass Transfer.24, (9). pp.1541-1544.(1927)

7. Lienhard J.H, A heat transfer textbook.-3 ${ }^{\text {rd }}$ ed.-Cambridge, M A: Phlogiston Press,. p. 749 .(2003).

8. Karman Th, The analogy between fluid friction and heat transfer.Trans.Amer.Soc.Mech.Engnr., 61, pp. 705-710. (1939)

9. Bradshaw P., Ferris D. H., Atwell N. P, Calculation of boundary layer development using the turbulent energy equation”, J. Fluid Mech. (1967).

10. Anderson D., Tannehil Doyu., Pletcher R, Computational fluid mechanics and heat transfer.- M.: Mir,. 1, (2), pp 384- 392. (1990)

11. A Spalart P.R., Shur, M.L, "On the sensitization of turbulence models to rotational and curvature". Aerospace Science and Technology. 1, (5), pp 297-302. (1997).

12. Prandtl L, Untersuchungen zur ausgebildete Turbulenz, Zeitschr. f. angew. Math. u. Mech. 5. (1925).

13. Libby, P. A. On the prediction of intermittent turbulent flows. Journal of Fluid Mechanics, 68 (2), pp 273 - 295. (1975).

14. Malin M.R., Spalding D.B, A two-fluid model of turbulence and its application to heated plane jets and wakes. J. Physico-chemical Hydrodyn. 5, pp. 339 - 362. (1984).

15. Yu K.Z. Ding G.L. Chen T.J, Modified two-fluid model for air curtains in open vertical display cabinets. Int. J. Refrig. 31, pp. 472 - 482, (2008)

16. F. Tachibana, S. Fukui, H. Mitsumura. Heat transfer in an annulus with inner rotating cylinder, Bulletin of JSME. 3 pp.119-123. (1960)

17. F. Tachibana, S. Fukui, Convective heat transfer of the rotational and axial flow between two concentric cylinders, Bulletin of JSME 7 , pp. 385-391. (1964).

18. M.M.M. El Telbany, A.J. Reynolds, The structure of turbulent plane Couette flow. J. of Fluids Engineering, 104, pp. 367 - 372. (1982)

19. Raleigh L. On the dynamics of revolving fluids. Proc. Roy. Soc. London, Ser. A, 93.(1916)

20. J.M. Robertson, On turbulent plane Couette flow. Proc. 6th Midwestern Conf. Fluid Mech., Univ. Texas, Austin, pp. 169-182. (1959).

21. Dopazo, C, On conditioned averages for intermittent turbulent flows. Journal of Fluid Mechanics, 81 (3), p 433 (1978)

22. Duhamel, $\mathrm{P}, \mathrm{A}$ detailed derivation of conditioned equations for intermittent turbulent flows, Letters in Heat and Mass Transfer, 8, pp. 491 - 502, (1978). 